\title{
TRAZAS FÓSILES DE NUCULOIDEOS (Protovirgularia) DEL MIOCENO MARINO DE LA CUENCA DEL VALLÈS-PENEDÈS
}

\author{
Jordi M. de GIBERT y Rosa DOMÈNECH \\ Departament d'Estratigrafia, Paleontologia i Geociències Marines. Universi- \\ tat de Barcelona. Martí Franquès s/n. 08028 Barcelona.jmdegibert@ub.edu, \\ rosa.domenech@ub.edu
}

\begin{abstract}
Gibert, J. M. de \& Domènech, R. 2008. Trazas fósiles de nuculoideos (Protovirgularia) del Mioceno marino de la Cuenca del Vallès-Penedès. [Nuculoidean trace fossils (Protovirgularia) from the marine Miocene of the Vallès-Penedès Basin.] Revista Española de Paleontología, 23 (2), 129-138. ISSN 0213-6937.
\end{abstract}

\begin{abstract}
The bivalve trace fossil Protovirgularia is described from Langhian (middle Miocene) shallow marine deposits of the Vallès-Penedès Basin in NE Spain. Three morphotypes have been defined. Morphotype 1 and 2 are negative epireliefs with a characteristic chevroned pattern and can be assigned to the ichnospecies P. dichotoma. The chevrons are the result of the activity of the cleft foot of a protobranch. Morphotype 3 ( $P$. cf. dichotoma) is preserved as epichnial backfilled full relieves, in occasions seen on top of morphotypes 1 and 2 . It may correspond to the trace left by the body of the animal when ploughing through the sediment. The tracemaker was most likely a nuculacean, as these bivalves are actively mobile deposit-feeders while other protobranchs are less active. In the Vallès-Penedès, several species of Nuculidae and Nuculanidae are known, which may have produced Protovirgularia. Nevertheless, they are normally of smaller size than expected for the tracemaker and found in different, although contemporaneous, facies.
\end{abstract}

Key words: Bivalvia, bioturbation, ichnology, Protovirgularia, Miocene, Vallès-Penedès.

\section{RESUMEN}

Se describe Protovirgularia, una pista de bivalvo, del Langhiense (Mioceno medio) de la Cuenca del Vallès-Penedès en el noroeste de España. Se distinguen tres morfotipos. Los morfotipos 1 y 2 son epirrelieves negativos con un característico patrón en espiga y pueden asignarse a la icnoespecie $P$. dichotoma. La estructura en espiga es el resultado de la actividad del pie bífido de un protobranquio. El morfotipo 3 ( $P$. cf. dichotoma) se conserva como relieves completos con retrorrelleno, a techo de capas de areniscas. En ocasiones, se observa su posición sobre pistas de los otros dos tipos. Estos relieves completos pueden corresponder a la traza dejada por el paso del cuerpo del animal. El productor de Protovirgularia fue muy probablemente un nuculáceo, ya que este grupo de bivalvos sedimentívoros son muy móviles a diferencia de otros protobranquios. En el Vallès-Penedès, se han identificado varias especies de nucúlidos y nuculánidos que podrían haber producido este tipo de pistas. Sin embargo, en general, son de talla menor a la que correspondería para el productor de las trazas descritas y, además, aparecen en otro tipo de facies.

Palabras clave: Bivalvos, bioturbación, icnología, Protovirgularia, Mioceno, Vallès-Penedès.

\section{INTRODUCCIÓN}

Los bivalvos son componentes fundamentales de la infauna y epifauna actual tanto en medios marinos como de agua dulce. Su papel como miembros de las comunidades bentónicas se remonta al Paleozoico inferior y es particularmente importante en medios marinos desde principios del Mesozoico (e.g., Fraisier \& Bottjer, 2007). El registro icnológico de este grupo de molus- cos es diverso e incluye tanto estructuras de bioerosión como de bioturbación. El icnogénero más frecuente en substrato duro es Gastrochaenolites Leymerie, 1842 que designa perforaciones en forma de ampolla realizadas en substratos minerales (líticos o biogénicos; ver Kelly \& Bromley, 1984). Otras perforaciones de habitación (Domichnia) incluyen los icnogéneros Petroxestes Wilson \& Palmer, 1988, Phrixichnus Bromley \& Asgaard, 1993, y Teredolites Leymerie, 1842, ésta última apareciendo so- 
lamente en substratos xílicos (de madera). Además, se han asignado a bivalvos algunas marcas de fijación (Fixichnia) como Centrichnus eccentricus Bromley \& Martinell, 1991, o Lacrimichnus bonarensis Santos, Mayoral $\&$ Muñiz, 2003. Por lo que respecta a las estructuras de bioturbación, diversos icnogéneros han sido atribuídos a bivalvos como Hillichnus Bromley, Uchman, Gregory \& Martin, 2003, Saronichnus Pervesler \& Zuschin, 2004, o Solemyatuba Seilacher, 1990, pero, sin lugar a dudas, los dos icnogéneros más frecuentes son Lockeia James, 1879, y Protovirgularia M'Coy, 1850. Lockeia (=Pelecypodichnus Seilacher, 1953) designa hiporrelieves positivos con morfología ovalada con los extremos en forma ojival y corresponde a estructuras de reposo $(\mathrm{Cu}-$ bichnia) (Seilacher \& Seilacher, 1994). Protovirgularia es una pista horizontal con un patrón interno muy característico formado por la repetición de una estructura en espiga (chevron). Se asigna al desplazamiento (Repichnia) de bivalvos protobranquios vágiles en el interior del sedimento.

Este artículo describe nuevo material de Protovirgularia procedente de los depósitos marinos del Langhiense (Mioceno medio) de la Cuenca del Vallès-Penedès en Cataluña, que constituye la primera cita de este icnogénero en el Neógeno de la Península Ibérica. Se analizan los posibles organismos productores de las pistas a partir del registro esquelético de bivalvos conocido para la cuenca y se discuten posibles variaciones morfológicas en el material estudiado en relación al proceso de formación y a condicionantes tafonómicos.

\section{MARCO GEOLÓGICO}

La Cuenca del Vallès-Penedès es una semifosa inscrita dentro de las Cadenas Costeras Catalanas o Catalánides. $\mathrm{Su}$ origen y formación está relacionado con la extensión que dió lugar al denominado Surco de Valencia, desarrollado durante el Neógeno entre la Península Ibérica y las Islas Baleares (Fontboté et al., 1990; Roca et al., 1999). Se trata de una cuenca estrecha (12-14 km) que se extiende más de $100 \mathrm{~km}$ en dirección SO-NE paralelamente a la costa (Fig. 1). Su relleno sedimentario está compuesto por más de $3000 \mathrm{~m}$ (en la zona NO donde se hallaba la falla activa) de depósitos continentales interrumpidos por tres unidades marinas principales correspondientes a las transgresiones del Burdigaliense superior, Langhiense y Serravaliense (Cabrera et al., 1991, 2004; Cabrera \& Calvet, 1996). Los icnofósiles aquí estudiados proceden de la unidad marina Langhiense que corresponde a la única transgresión que alcanzó el sector más interno de la cuenca en la zona de El Vallès. En concreto, Protovirgularia aparece en depósitos heterolíticos formados en zonas distales de abanicos costeros alimentados por los relieves del margen NO de la cuenca.

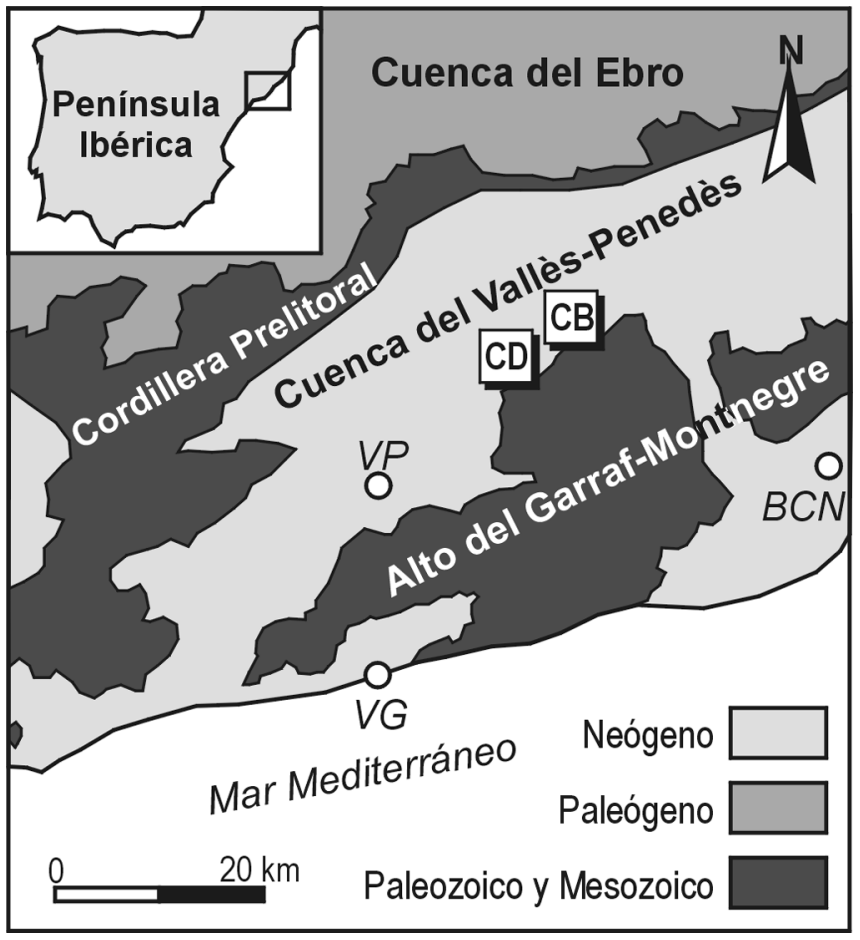

Figura 1. Mapa geológico sintético de la Cuenca del VallèsPenedès, modificado de Cabrera et al. (1991). Se indica la situación de los afloramientos con Protovirgularia $(\mathrm{CD}=\mathrm{Bosc}$ de Can Duran, $\mathrm{CB}=\mathrm{Can}$ Bosch) y algunas poblaciones de referencia $(\mathrm{BCN}=$ Barcelona, $\mathrm{VP}=$ Vilafranca del Penedès, VG= Vilanova i la Geltrú).

Synthetic geological map of the Vallès-Penedès Basin, modified from Cabrera et al. (1991) with indication of the outcrops that yielded Protovirgularia $(C D=B o s c$ de Can Duran, $C B=C a n$ Bosch) and some cities for situation $(B C N=$ Barcelona, $V P=$ Vilafranca del Penedès, $V G=$ Vilanova $i$ la Geltrú).

\section{MATERIAL Y LOCALIDADES}

Los especímenes estudiados proceden de la zona conocida como Bosc de Can Duran, situada a aproximadamente $1,5 \mathrm{~km}$ al norte de la localidad de Gelida en la comarca del Alt Penedès (Fig. 1). En este sector la serie marina Langhiense está constituída por alternancias de areniscas y lutitas ocres, depositadas en zonas sumergidas de abanicos costeros, y por margas gris-azules, correspondientes a la sedimentación en zonas más distales. Estos depósitos terrígenos intercalan unidades de orden métrico constituídas por areniscas con gran contenido bioclástico (principalmente fragmentos de conchas de moluscos) que registran episodios transgresivos de menor orden. Protovirgularia aparece en la parte alta de un tramo siliciclástico de unos $20 \mathrm{~m}$ entre dos unidades transgresivas, a techo de capas de areniscas de 1-2 $\mathrm{cm}$ de grosor intercaladas en lutitas ocres. La asociación de trazas fósiles se completa con $\mathrm{Pa}$ - 

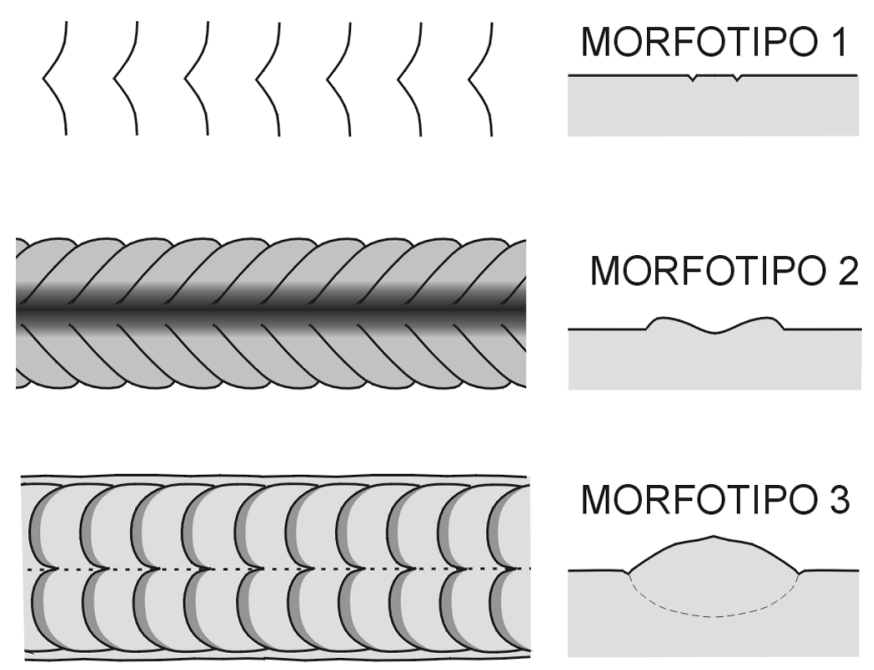

Figura 2. Representación esquemática (vista superior y corte transversal) de los tres morfotipos de Protovirgularia.

Schematic drawing (upper view and cross section) of the three morphotypes of Protovirgularia.

laeophycus Hall, 1847, Bichordites Plaziat \& Mahmoudi, 1988, y Planolites Nicholson, 1873. Ademàs se ha obtenido una losa con varios especímenes de Protovirgularia y Bichordites en la localidad de Can Bosch, a unos $3 \mathrm{~km}$ al SO de la primera en un contexto estratigráfico muy similar aunque en niveles algo inferiores.

Los especímenes recolectados se hallan depositados en la colección de icnología del Departament d'Estratigrafia, Paleontologia i Geociències Marines de la Universitat de Barcelona, con los números de registro: IC-356, IC-379, IC-380 y IC-433 a IC-436.

\section{DESCRIPCIÓN DE LAS TRAZAS FÓSILES}

El conjunto de trazas fósiles descritas aquí son comparables a diversos ejemplos publicados de Protovirgularia (véase la sección "discusión icnotaxonómica"), pero aún así presentan una variación morfológica importante. Esto nos ha llevado a caracterizar tres morfotipos (Fig. 2).

\section{Morfotipo 1}

Figs. 3a, g

Es el más simple y agrupa epirrelieves negativos constituídos por surcos poco profundos en forma de $\mathrm{V}$ muy laxa alineados a lo largo de un eje central. La anchura de estas pistas varía entre 4 y $5 \mathrm{~mm}$ mientras que la distancia entre surcos sucesivos oscila entre 2 y $3,5 \mathrm{~mm}$. Cada una de las dos mitades de las marcas en $\mathrm{V}$ se dispone con un ángulo cercano a los $75^{\circ}$ respecto al eje de la pista, y presenta una ligera convexidad opuesta al vértice en que se unen. En algunos casos, éste vértice puede estar ausente de manera que ambas mitades quedan separadas.

\section{Morfotipo 2}

Fig. 3c

Consiste en epirrelieves compuestos por un surco central y unos lóbulos laterales de forma linguoide dispuestos oblicuamente formando un ángulo de unos $60^{\circ}$ respecto al surco y en contacto uno con el otro. La anchura de la pista oscila entre 4,5 y 5 $\mathrm{mm}$, mientras que la de los lóbulos laterales (perpendicularmente a su eje longitudinal) es de aproximadamente $1 \mathrm{~mm}$.

\section{Morfotipo 3}

Figs. 3b, d-f, 4a, b

Esta forma es la más abundante de las tres. Se trata de relieves completos que aparecen a techo de las capas de areniscas. La sección es circular a oval con una anchura entre 4 y 6 $\mathrm{mm}$. Internamente presentan un retrorrelleno (backfill) en forma de paquetes discretos de sedimento arenoso. Éstos pueden tener una morfología irregular pero en la mayoría de los casos están limitados por superficies biconvexas. El grosor de dichos paquetes oscila entre 1 y $3,5 \mathrm{~mm}$ y puede variar dentro de una misma pista. En algunos casos se observa un fino manto alrededor de la pista.

La relación entre éste y los otros morfotipos se constata en algunos especímenes. En un caso (Fig. 4a, b) se observa una forma transicional entre un surco correspondiente al morfotipo 2 y un relleno completo del morfotipo 3. En otro ejemplar (Fig. 3b), que corresponde al tercer morfotipo, se reconocen unas marcas laterales que recuerdan a las estructuras en $\mathrm{V}$ de los morfotipos 1 y 2 . Así pues, parece que pese a las marcadas diferencias, los morfotipos corresponden a distintas conservaciones de una misma estructura.

\section{Posibles trazas de reposo asociadas}

En otros ejemplos descritos, es común que Protovirgularia aparezca asociada a trazas de reposo de tipo Lockeia, de morfología ovalada con los extremos apuntados (e.g., Mángano et al., 1998; Ekdale \& Bromley, 2001). En el material estudiado, se han reconocido algunas estructuras pobremente conservadas con morfologías que recuerdan a Lockeia (Figs. 3a, 4c, d) aparentemente asociadas con algunas pistas a techo de las capas de arenisca. La toponimia de estos especímenes de Lockeia contrasta con la mayor parte del material descrito previamente por otros autores (e.g., Mángano et al., 1998) que típicamente corresponde a hiporrelieves positivos. Sin embargo, también se conocen Lockeia conservadas en epirrelieve (Mángano et al., 2002).

\section{DISCUSIÓN ICNOTAXONÓMICA}

El icnogénero Protovirgularia ha sido objeto de diversas revisiones desde el punto de vista icnotaxonómico. Para Han \& Pickerill (1994) este icnogénero designaba únicamente semirrelieves en forma de quilla con marcas en forma de espiga (chevron). Sin embargo, tanto Seilacher \& 

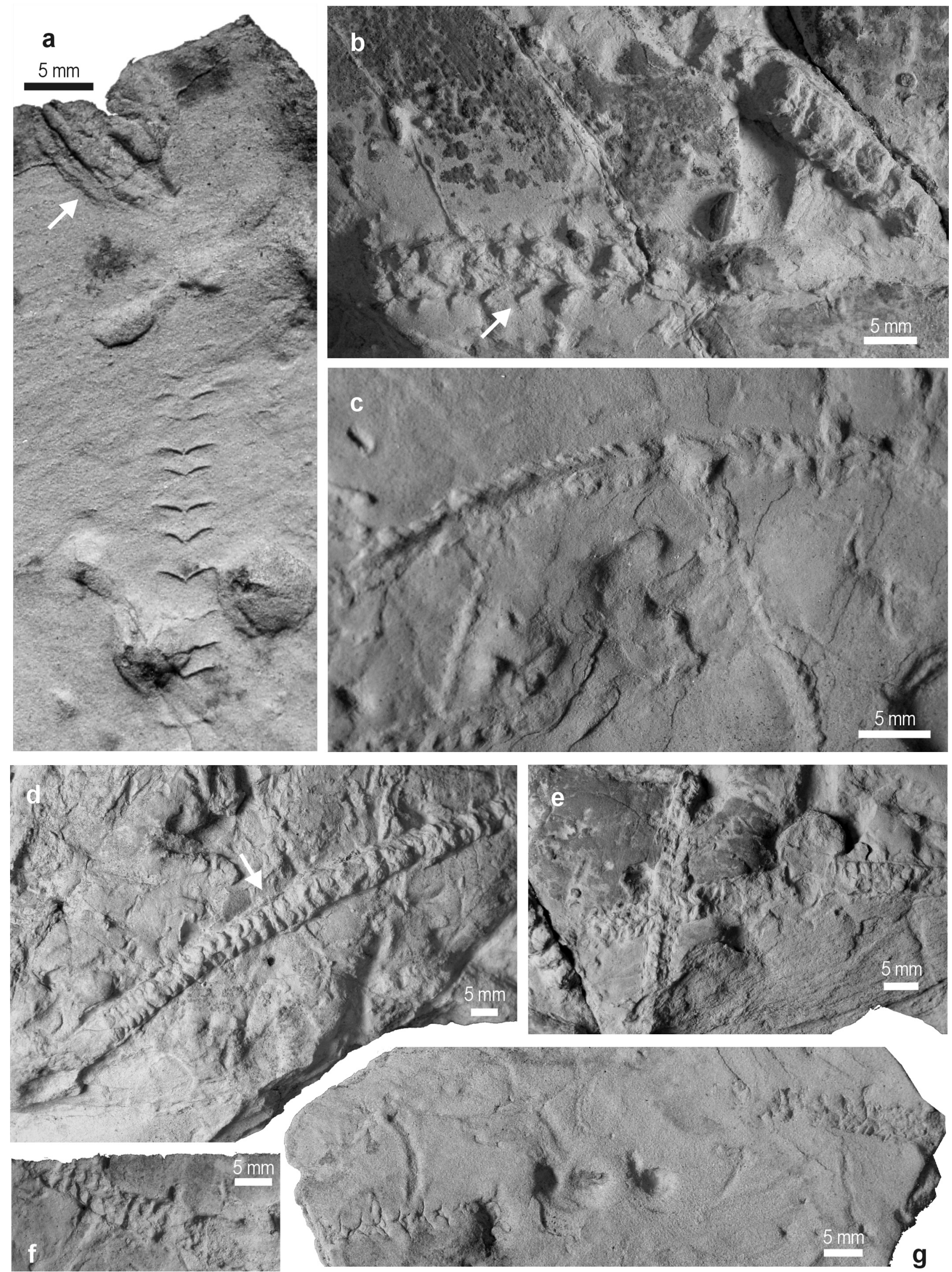
Seilacher (1994) como Uchman (1998) ampliaron la diagnosis para incluir también estructuras en relleno completo. Estos mismos autores, así como Mángano et al. (1998), consideraron Chevronichnus Hakes, 1976, Imbrichnus Hallam, 1970, Sustergichnus Chamberlain, 1971, Uchirites Macsotay, 1967, y Walcottia Miller \& Dyer, 1878 como sinónimos subjetivos más recientes de Protovirgularia.

La asignación de los morfotipos 1 y 2 a Protovirgularia es clara ya que ambos presentan la característica estructura en espiga. El morfotipo 2 es muy comparable a los especímenes descritos por Hakes (1976) en la definición de la icnoespecie Chevronichnus imbricatus. Sin embargo, esta icnoespecie debe ser considerada como un sinónimo moderno de Protovirgularia dichotoma McCoy, 1850. Tanto el material de Hakes (1976) como el que aquí se describe son epirrelieves negativos mientras que la mayor parte de otros ejemplos de P. dichotoma son hiporrelieves positivos (e.g. Ekdale \& Bromley, 2001). Sin embargo, esta diferencia en la conservación no debería tener consecuencias taxonómicas. De hecho, Han \& Pickerill (1994) incluyeron tanto hiporrelieves positivos como epirrelieves negativos en su revisión de la icnoespecie. Por lo que respecta al morfotipo 1 por su toponimia y morfología podría ser confundido con un rastro de artrópodo pero, como indican Seilacher \& Seilacher (1994), los artrópodos tienden a colocar sus extremidades de manera alternante (con la excepción de los limúlidos) por lo que los rastros no son bilateralmente simétricos. Tanto Han \& Pickerill (1994) como Mángano et al. (1998) incluyeron formas similares a nuestro morfotipo 1 en Protovirgularia dichotoma, asignación que compartimos en el caso del material del Penedès. Las diferencias entre los morfotipos 1 y 2 tienen que ver con la posición de la interfase arena/fango en relación con la actividad del organismo productor (como se discute más adelante) y por tanto no tiene consecuencias taxonómicas.

El morfotipo 3 es el que presenta más problemas de asignación icnotaxonómica. Diversos autores han descrito y figurado como Protovirgularia relieves completos muy similares a estas formas (e.g. Uchman, 1998; Mángano et al., 1998). Sin embargo, éstos son siempre descritos en bases de capas de manera que la morfología observable es la de la cara inferior de la pista. Los ejemplares del morfotipo 3 del Mioceno del Penedés aparecen a techo de areniscas y por tanto muestran la cara superior de la traza, o en algunos casos su estructura interna. La polaridad de las capas que contienen las pistas aquí descritas queda claramente definida por la presencia de marcas de base (tool marks) y por su laminación cruzada interna. Protovirgularia dzulynskii (Książkiewicz, 1977) designa relieves completos con una delgada pared y con relleno meniscado. Esta icnoespecie, originalmente atribuída con dudas al icnogénero Arthrophycus Hall, 1852, fue asignada por Uchman (1998) a Protovirgularia por su asociación con trazas de tipo Lockeia y por presentar en algunos casos un patrón en espiga. Presenta algunas similitudes con nuestro morfotipo 3 aunque en éste último las crestas no son tuberculadas, una de las características diagnósticas de P. dzulynskii. Por otro lado, la asociación vista entre los morfotipos 2 y 3 (Fig. 4a, b) sugiere que ambos son parte de una misma estructura. Por ello, preferimos aquí designar los especímenes del morfotipo 3 como Protovirgularia cf. dichotoma.

\section{MECANISMO DE FORMACIÓN Y ORGANISMO PRODUCTOR}

Protovirgularia es reconocida hoy en día como una traza fósil producida por la actividad de bivalvos endobentónicos (Han \& Pickerill, 1994; Seilacher \& Seilacher, 1994; Mángano et al., 1998). Su típica asociación en continuidad con Lockeia supone un argumento sólido para esta asignación. Así mismo, Seilacher \& Seilacher (1994) realizaron un estudio experimental con bivalvos actuales y obtuvieron pistas asignables a Protovirgularia como resultado de la actividad de nuculoideos de los géneros Acila Adams \& Adams, 1858, y Yoldia Möller, 1842.

\section{Mecanismos de excavación en los bivalvos}

Existen numerosos estudios que analizan los sistemas de excavación de los bivalvos endobentónicos, así como su relación con la morfología de la concha (Trueman, 1966, 1968a; Trueman et al., 1966; Stanley, 1970, 1975). El mecanismo utilizado por estos moluscos para penetrar en el sedimento y moverse a través de él fue descrito en

Figura 3. Protovirgularia del Mioceno del Penedès. Todos los ejemplares proceden de Bosc de Can Duran excepto c que procede de Can Bosch. En todos los casos las trazas están a techo. a, Espécimen del morfotipo 1 asociado a Lockeia (flecha); IC434. b, Dos ejemplares en relieve completo (morfotipo 3), uno de ellos presentando marcas laterales oblicuas (flecha); IC-436. c, Ejemplares del morfotipo 2; IC-4256. d, Espécimen del morfotipo 3 que muestra un manto externo (flecha); IC-380. e, Dos ejemplares mostrando la estructura interna del morfotipo 3; IC-436. f, Otro ejemplo del morfotipo 3; IC379. g, Especímenes del morfotipo 1; IC-433.

Miocene Protovirgularia from the Penedès. All material from Bosc de Can Duran except for c that comes from Can Bosch. All pictures show the top of the sandstone beds. a, Morphotype 1 associated to Lockeia (arrow); IC-434. b. Two specimens in full relief (morphotype 3), one of them displaying lateral oblique markings (arrow); IC-436. c, Specimens of morphotype 2; IC-256. d, Morphotype 3 displaying the external mantle (arrow); IC-380. e, Two specimens showing the internal structure of morphotype 3; IC-436.f, Another example of morphotype 3; IC-379. g, Two specimens of morphotype 1; IC-433. 
detalle por Trueman (1966; ver también Seilacher \& Seilacher, 1994). Este sistema se enmarca en una técnica habitual en invertebrados bioturbadores conocida como mecanismo de doble ancla (Bromley, 1990) o de push-andpull (empujar y estirar). La excavación se lleva a cabo mediante una serie de movimientos que implican todo el sistema muscular. El desplazamiento se inicia mediante la extensión del pie musculoso entre las valvas para penetrar en el substrato. Las propias valvas actuan como una primera ancla que permite esa penetración del pie. Después, la sangre es bombeada al interior del pie, lo que causa que se hinche y actúe como una segunda ancla en el sedimento. Entonces los músculos longitudinales se contraen acortando el pie y tiran del animal hacia delante. Este ciclo se repite sucesivamente hasta que el animal alcanza la posición deseada.

La morfología del pie de los bivalvos responde a dos patrones básicos. La mayoría de bivalvos (los polisiringios) presentan un pie cuneiforme (wedge foot), especializado en la excavación. El segundo patrón es el pie bífido (cleft foot), que aparece en los bivalvos protobranquios y en la superfamília Arcacea. Aunque aparentemente se halla en grupos primitivos, la funcionalidad de este pie es diversa ya que, además de su papel como órgano excavador, también sirve como sistema de anclaje en el interior del sedimento. La función del pie bífido se ha estudiado en el arcáceo Glycymeris Da Costa, 1778 (ver Ansell \& Trueman,1967). En la fase de penetración en el sedimento los dos pliegues se cierran para formar una cuña. A continuación, se abren para formar una eficiente ancla. Éste mecanismo explica bien la forma en espiga de las trazas internas (undertraces) que dejan estos animales, como se ha comprobado experimentalmente y se observa en las trazas fósiles (Seilacher \& Seilacher, 1994). Otro grupo de moluscos endobentónicos con pie excavador bífido y que pueden desplazarse dentro del sedimento es el de los escafópodos (Trueman, 1968b; Seilacher \& Seilacher, 1994). Sin embargo, parece que no acostubran a cambiar de ubicación o lo hacen de forma muy puntual (Yochelson, 1999) por lo que no es probable que generen pistas largas y continuas.
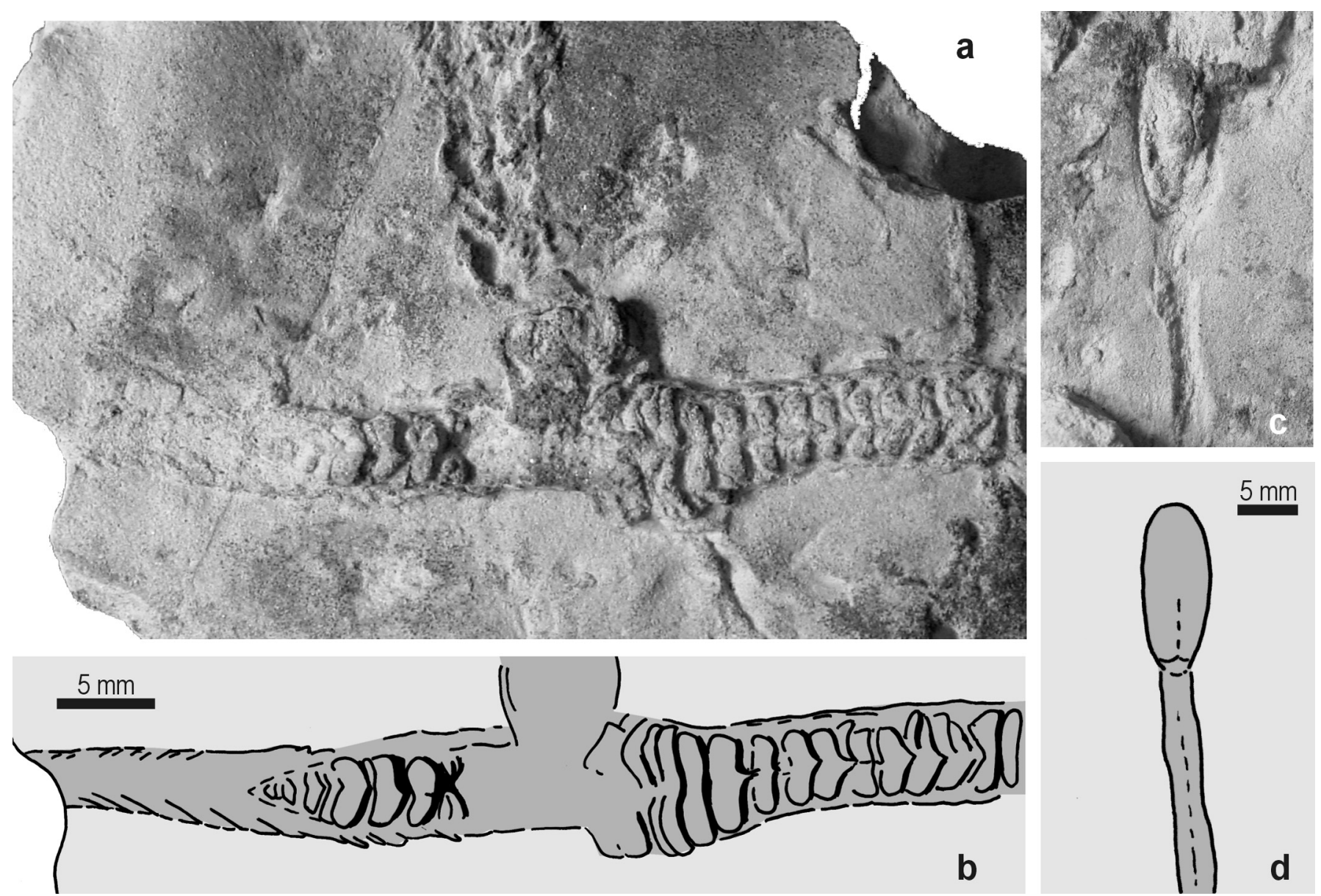

Figura 4. a-b, Ejemplar transicional entre un epirrelieve negativo con estructura en espiga (morfotipo 2, izquierda) y una forma en relieve completo (morfotipo 3, derecha); IC-435. c-d, Lockeia asociada a una pista en epirrelieve negativo; IC-435.

$\boldsymbol{a}-\boldsymbol{b}$, Transitional specimen between a chevroned negative epirelief (morphotype 2, left) and a full relief form (morphotype 3, right); IC-435. c-d, Lockeia associated to a negative epirelief trail; IC-435. 


\section{Formación de las pistas del Penedès}

Los especímenes de los morfotipos 1 y 2 definidos en el material del Penedès son muy similares a las pistas obtenidas experimentalmente por Seilacher \& Seilacher (1994). Por tanto, pueden interpretarse como trazas endogénicas producidas por el pie bífido de un bivalvo protobranquio. El patrón en espiga resultaría del movimiento de separación de las dos partes del pie para fijar el extremo anterior del animal al substrato. La superficie de colonización se situaría por encima del techo de la capa arenosa de manera que la traza dejada por el paso del cuerpo (y la concha) del molusco se habría producido en los fangos suprayacentes y no habría quedado conservada. El morfotipo 1 probablemente representa situaciones en que se dió un menor contacto entre el pie y el depósito arenoso que en el morfotipo 2, quizás debido a un grosor superior de los fangos suprayacentes.

El morfotipo 3 resulta, en cambio, más difícil de interpretar. Estas formas parecen poseer un retrorrelleno, carácter que si bien ha sido descrito para algunas icnoespecies fósiles ( $P$. dzulynskii) no fue observado por Seilacher \& Seilacher (1994). El diseño de su experimento desveló la pista dejada por el pie bifurcado como un molde de la impresión dejada sobre un fango enterrado en arena, es decir en forma de semirrelieve, nunca de relieve completo. Parece difícil explicar la formación del relieve completo únicamente a partir de la actividad del pie bifurcado. La existencia de especímenes transicionales o combinados entre el morfotipo 2 y el 3 aporta nuevos datos para entender este tipo de relieves completos. En estos ejemplares (Figs. 3b, 4a, b) se observa un relieve completo (morfotipo 3) bajo el cuál se reconoce un epirrelieve negativo con estructura en espiga (morfotipo 2). Ésto nos lleva a considerar que, si la parte inferior corresponde a la marca del pie, el relieve completo podría corresponder a la traza dejada por el paso del resto del animal con su concha. La transición entre ambos morfotipos indicaría un ligero y progresivo cambio en la profundidad de bioturbación respecto al techo de la capa de areniscas. Aunque se considera normalmente que la concha no deja ninguna estructura reconocible (Seilacher \& Seilacher, 1994), el retrorrelleno observado podría interpretarse como una sucesión de spreiten indicadores de anteriores posiciones de la concha. En este caso, cuando la concha es estirada anteriormente dejaría un espacio que se rellenaría pasivamente de sedimento, el cual, en el "paso" siguiente, podría ser compactado por la presión ejercida por la concha como reacción a la nueva penetración del pie en el sedimento. La inflexion axial del retrorelleno podría tener que ver con la morfología bivalvada de la concha. Esta hipótesis explicaría la existencia de rellenos completos, aunque su confirmación queda pendiente de la realización de nuevos experimentos y/o de la observación de más ejemplares.

\section{El organismo productor}

Los experimentos de Seilacher \& Seilacher (1994) muestran la capacidad de los nuculoideos para producir pistas en espiga del tipo Protovirgularia. De todas maneras, el pie bifurcado responsable de este particular patrón existe en otros protobranquios, en la superfamilia Arcacea y en los escafópodos.

Los escafópodos pueden ser descartados como productores de las trazas del Mioceno del Penedès en base a tres argumentos: 1) la asociación de Protovirgularia con estructuras de tipo Lockeia (Figs. 3a, 4c, d) apunta a un productor bivalvo; 2) los escafópodos son menos activos y tienden a permanecer más tiempo en una misma ubicación, y 3) a pesar del extenso registro de moluscos en la cuenca del Vallès-Penedès los escafópodos sólo aparecen de manera muy local en algunas localidades.

Así pues, el productor de Protovirgularia tuvo que ser un bivalvo altamente móvil. Entre los bivalvos endobentónicos hay una gran mayoría de especies sésiles suspensívoras, mientras que sólo unas pocas son detritívoras y vágiles. Éste hábito está hoy en día restringido a las superfamilias Nuculacea y Tellinacea. Éstos últimos no son protobranquios ni presentan un pie bífido y así pueden ser descartados como productores de Protovirgularia, aunque sí que pueden ser responsables de otras trazas fósiles (Bromley et al., 2003). Por ello, un nuculáceo (familias Nuculidae y Nuculanidae) parece el candidato más adecuado para ser considerado el autor de Protovirgularia. Los nuculáceos recolectan el alimento del sedimento mediante las probóscides, que son apéndices de los palpos labiales, aunque no todos se alimentan siguiendo el mismo patrón. Así, Nucula normalmente come justo por encima de la superficie del fondo, mientras que Yoldia puede mantener el tercio posterior del cuerpo desenterrado de manera que las palpos recolectan las partículas alimentarias de la superficie (Purchon, 1968).

El registro malacológico del Mioceno Vallès-Penedès es extenso y conocido ya desde el siglo XIX (Almera, 1896; Crusafont, 1959; Martinell \& Porta, 1981; Navas, 1991; Batllori \& Martinell, 1993; Batllori, 1995). Este registro incluye material perteneciente a diversas especies de bivalvos protobranquios. Hasta el momento los estudios en curso nos han permitido identificar las siguientes especies: Nucula nitidosa Winckworth, 1930, N. placentina (Lamarck, 1819), Nuculana (Lembulus) emarginata (Lamarck, 1819), $N$. (L.) pella (Linné, 1767) y Ledella aff. messanensis (Jeffreys, 1870), así como dos especies más todavía no determinadas de Ledella Verrill \& Bush, 1897 y Nucula Lamarck, 1799, y diversas de Nuculana Link, 1807. Se trata, en conjunto, de ejemplares de pequeña talla respecto a la mediana de estas formas, en especial por lo que se refiere a Nucula. Los ejemplares de nuculoideos proceden de la unidad de margas gris-azuladas que corresponden a depósitos más distales y profundos que las unidades heterolíticas en las que aparece Protovirgularia. En 
éstas, por cuestiones diagenéticas, es muy raro encontrar fósiles de esqueletos aragoníticos. Por otro lado, los especímenes encontrados de las especies aquí mencionadas son de dimensiones mucho menores a las esperables para los productores de las pistas. Así pues, resulta imposible determinar el productor de las mismas más allá de que se trata de un nuculáceo.

\section{CONCLUSIONES}

Las trazas fósiles descritas en este trabajo constituyen el primer registro de Protovirgularia en el Neógeno ibérico. El material estudiado presenta una variación morfológica debida a cuestiones tafonómicas, en concreto a la posición del productor respecto a la interfase arena/ fango. Esta circunstancia ha permitido la caracterización de tres morfotipos distintos. Así, los morfotipos 1 y 2, asignables a Protovirgularia dichotoma, registran solamente la actividad del pie muscular bífido del bivalvo productor que resulta en epirrelieves negativos con estructura en espiga. El morfotipo 3 (Protovirgularia cf. dichotoma), en cambio, se conserva como rellenos completos con retrorrelleno que se superponen a estructuras en espiga. Estos rellenos completos podrían registrar el paso de la concha del animal aunque más material y nuevos estudios experimentales son necesarios para confirmar este punto. La particular conservación de Protovirgularia en el techo de capas de areniscas no es una novedad pero si una rareza puesto que este icnogénero se encuentra normalmente como hiporrelieves. Su posición toponímica indica colonización desde los fangos suprayacentes depositados tras la sedimentación de las areniscas probablemente como la parte terminal del proceso turbidítico.

Las pistas son atribuídas a bivalvos nuculáceos en base a su asociación con Lockeia, a la comparación con estudios experimentales y a datos conocidos sobre el hábito de vida de estos bivalvos. Sin embargo, y a pesar de tener un registro abundante de nuculáceos en la cuenca no es posible precisar más, puesto que los fósiles corporales aparecen en facies margosas correspondientes a paleoambientes más distales y profundos que las facies heterolíticas en que aparecen las trazas. En éstas últimas los procesos diagenéticos no han permitido la conservación de conchas aragoníticas.

\section{AGRADECIMIENTOS}

Los autores agradecen a Nuria Martínez Lázaro la colaboración en la determinación de los nuculáceos y a Jordi Martinell por sus valiosos comentarios. Este trabajo forma parte de las actividades el proyecto de investigación CGL 2007-60507/ BTE del Ministerio de Educación y Ciencia.

\section{REFERENCIAS}

Adams, H. \& Adams, A. 1858. The genera of recent Mollusca arranged according to their organization. Van Voorst, Londres, 1283 pp.

Almera, J. 1896. Reconocimiento de la presencia del primer piso mediterráneo en el Panadés. Memorias de la Real Academia de Ciencias y Artes de Barcelona, 1, 349394.

Ansell, A.D. \& Trueman, E.R. 1967. Observations on burrowing in Glycymeris glycymeris (L.) (Bivalvia, Arcaea). Journal of Experimental Marine Biology and Ecology, 1, 65-75.

Batllori, J. 1995. Gasterópodos del Mioceno medio marino del Alt Penedès (Catalunya). Tesis doctoral, Universitat de Barcelona, 591 pp. (inédita).

Batllori, J. \& Martinell, J. 1993. Malacofauna del Mioceno salobre de Cerdanyola del Vallès (Catalunya): aspectos paleoecológicos. Iberus, 11, 1-8.

Bromley, R.G. 1990. Trace fossils. Biology and taphonomy. Unwin Hyman, Londres, 280 pp.

Bromley, R.G. \& Asgaard, U. 1993. Endolithic community replacement on a Pliocene rocky coast. Ichnos, 2, 93-116.

Bromley, R.G. \& Martinell, J. 1991. Centrichnus, new ichnogenus for centrically patterned attachment scars on skeletal substrates. Bulletin of the Geological Society of Denmark, 38, 243-252.

Bromley, R.G., Uchman, A., Gregory, M. \& Martin, A. 2003. Hillichnus lobosensis igen. et isp. nov., a complex trace fossil produced by tellinacean bivalves, Paleoecene, Monterey, California, USA. Palaeogeography, Palaeoclimatology, Palaeoecology, 192, 157-186.

Cabrera, L. \& Calvet, F. 1996. Onshore Neogene record in NE Spain: Vallès-Penedès and El Camp half-grabens (NW Mediterranean). In: Tertiary basins of Spain (Eds. P.F. Friend \& C.J. Dabrio). Cambridge University Press, Cambridge, 97-105.

Cabrera, L., Calvet, F., Guimerà, J. \& Permanyer, A. 1991. El registro sedimentario miocénico en los semigrabens del Vallès-Penedès y de El Camp: organización secuencial y relaciones tectónica sedimentación. In: Libro-guía excursión no. 4, I Congreso del Grupo Español del Terciario (Ed. F. Colombo). Universitat de Barcelona, Barcelona, 1-132.

Cabrera, L., Roca, E., Garcés, M. \& Porta, J. de, 2004. Estratigrafía y evolución tectonosedimentaria oligocena superior-neógena del sector central del margen catalán (Cadena Costero Catalana). In: Geología de España (Ed. J.A. Vera). Sociedad Geológica de España - Instituto Geológico y Minero de España, Madrid, 569-573.

Chamberlain, C.K. 1971. Morphology and ethology of trace fossils from the oauchita Mountains. Journal of Paleontology, 45, 212-246.

Crusafont, M. 1959. La segunda fase transgresiva en el Vindoboniense del Vallés-Penedés. Notas y Comunicaciones, Instituto Geológico y Minero de España, 55, 3-15.

Da Costa, E.M. 1778. Historia naturalis testaceorum Britannice. Millan, White, Emsley \& Robson, Londres, 254 pp. 
Ekdale, A.A. \& Bromley, R.G. 2001. A day and a night in the life of a cleft-foot clam: Protovirgularia-LockeiaLophoctenium. Lethaia, 34, 119-124.

Fontboté, J.M., Guimerà, J., Roca, E., Sàbat, F., Santanach, P. \& Fernández-Ortigosa, F. 1990. The Cenozoic geodynamic evolution of the Valencia Trough (Western Mediterranean). Revista de la Sociedad Geológica de España, 3, 249-259.

Fraisier, M.L. \& Bottjer, D.J. 2007. When bivalves took over the world. Paleobiology, 33, 397-413.

Hakes, W.G. 1976. Trace fossils and depositional environment of four clastic units, Upper Pennsylvanian megacyclothems, northeast Kansas. The University of Kansas Paleontological Contributions, 63, 1-46.

Hall, J. 1847. Paleontology of New York. Volume 1. State of New York, Albany, 338 pp.

Hall, J. 1852. Paleontology of New York. Volume 2. State of New York, Albany, 362 pp.

Hallam, A. 1970. Gyrochorte and other trace fossils in the Forest Marble (Bathonian) of Dorset, England. In: Trace fossils, Geological Journal Special Issue 3 (Ed. T.P. Crimes \& C. Harper). Seel House Press, Liverpool, 189-200.

Han, Y. \& Pickerill, R.K. 1994. Taxonomic reassessment of Protovirgularia M'Coy 1850 with new examples from the Paleozoic of New Brunswick, eastern Canada. Ichnos, 3, 202-212.

James, U.P. 1879. Description of new species of fossils and remarks on some others, from the Lower and Upper Silurian rocks of Ohio. The Paleontologist, 3, 17-24.

Jeffreys, J.G. 1870. Mediterranean Mollusca. Annals and Magazine of Natural History, Serie 4, 6, 65-86.

Kelly, S.R.A. \& Bromley, R.G. 1984. Ichnological nomenclature of clavate borings. Palaeontology, 27, 793-807.

Książkiewicz, M. 1977. Trace fossils in the flysch of the Polish Carpathians. Palaeontologica Polonica, 36, 1-208.

Lamarck, J.B. 1799. Prodrome d'une nouvelle classification des coquilles, comprenant une rédaction appropriée des caractères génériques, et l'établissement d'un grand nombre de genres nouveaux. Societé d'Histoire Naturelle Paris, Mémoir, 1, 63-91.

Lamarck, J.B. 1819. Histoire naturelle des animaux sans vertèbres. Tome 6. París, $343 \mathrm{pp}$.

Leymerie, M.A. 1842. Suite du mémoire sur le terrain Crétacé du Département de l'Aube. Societé Géologique de France, Mémoire, 5, 1-34.

Link, H.F. 1807. Beschreibung der Naturalien-Sammlung der Universität zu Rostock. Rostock, 160 pp.

Linné, C. von 1767. Systema Naturae. Ed. XII, I (Regnum Animale). Laurentii Salvii, Estocolmo, 533-1327.

M'Coy, F. 1850. On some genera and species of Silurian Radiata in the collection of the University of Cambridge. Annual Magazine of Natural History, 6, 270-290.

Macsotay, O. 1967. Huellas problematicas y su valor ecológico en Venezuela. Geos (Venezuela), 16, 7-79.

Mángano, M.G., Buatois, L.A., West, R.R. \& Maples, C.G. 1998. Contrasting behavioral and feeding strategies recorded by tidal-flat bivalve trace fossils from the Upper Carboniferous of Eastern Kansas. Palaios, 13, 335-351.
Mángano, M.G., Buatois, L.A., West, R.R. \& Maples, C.G. 2002. Ichnology of a Pennsylvanian equatorial tidal flats - the Stull Shale Member at Waverly, eastern Kansas. Bulletin of the Kansas Geological Survey, 245, 1-133.

Martinell, J. \& Porta, J. de 1981. Presencia de Vaginella austriaca KITTL (Pteropoda) y fauna malacológica acompañante en el Mioceno de Catalunya. Iberus, 1, 1-8.

Miller, S.A. \& Dyer, C.B. 1878. Contributions to paleontology, no. 1. Cincinnati Society of Natural History, Journal, 1, 24-39.

Möller, H.P.C. 1842. Index molluscorum Groenlandiae. Naturhistorisk Tiddskrift, 4, 76-97.

Navas, E., 1991. La malacofauna del yacimiento miocénico de Sant Pau d'Ordal (Barcelona): tafonomía y sistemática. Tesis de licenciatura, Universitat de Barcelona, 313 pp. (inédita).

Nicholson, H.A. 1873. Contributions to the study of the errant annelides of the older Paleozoic rocks. Royal Society London Proceedings, 21, 288-290.

Pervesler, P. \& Zuschin, M. 2004. A lucinoid bivalve trace fossil Saronichnus abeli igen. et isp. nov. from the Miocene molasse deposits of Lower Austria and its environmental significance. Geologica Carpathica, 55, 111-115.

Plaziat, J.C. \& Mahmoudi, M. 1988. Trace fossils attributed to burrowing echinoids: a revision including new ichnogenus and ichnospecies. Geobios, 21, 209-233.

Purchon, R.D. 1968. The Biology of the Mollusca. Pergamon Press, New York, 560 pp.

Roca, E., Sans, M., Cabrera, L. \& Marzo, M. 1999. Oligocene to Middle Miocene evolution of the central Catalan margin (northwestern Mediterranean). Tectonophysics, 315, 209-229.

Santos, A., Mayoral, E. \& Muñiz, F. 2003. New trace fossils produced by etching molluscs from the Upper Neogene of the southwestern Iberian Peninsula. Acta Geologica Polonica, 53, 181-188.

Seilacher, A. 1953. Studien zur Palichnologie. II. Die fossilien Ruhespuren (Cubichnia). Neues Jahrbuch für Geologie und Paläontologie, Abhandlungen, 98, 87-124.

Seilacher, A. 1990. Aberrations in bivalve evolution related to photo- and chemosymbiosis. Historical Biology, 3, 289-311.

Seilacher, A. \& Seilacher, E. 1994. Bivalvian trace fossils: a lesson from actuopalaeontology. Courier Forschungsinstitut Senckenberg, 169, 5-15.

Stanley, S.M. 1970. Relation of shell form to life habits of the Bivalvia (Mollusca). Geological Society of America Memoir, 25, 1-269.

Stanley, S.M. 1975. Why clams have the shape they have: an experimental analysis of burrowing. Palaeobiology, 7, 48-58.

Trueman, E.R. 1966. Bivalve molluscs: fluid dynamics of burrowing. Science, 152, 523-525.

Trueman, E.R. 1968a. The burrowing activities of bivalves. Symposia of the Zoological Society of London, 22, 176186.

Trueman, E.R. 1968b. The burrowing process of Dentalium (Scaphopoda). Journal of Zoology, London, 154, 19-27. 
Trueman, E.R., Brand, A.R. \& Davis, P. 1966. The dynamics of burrowing of some common littoral bivalves. Journal of Experimental Biology, 44, 469-492.

Uchman, A. 1998. Taxonomy and ethology of flysch trace fossils: revision of the Marian Książkiewicz collection and studies of complementary material. Annales Societatis Geologorum Poloniae, 68, 105-218.

Verrill, A.E. \& Bush, K.J. 1897. Revision of the genera of Ledidae and Nucilidae of the Atlantic Coast of the United States. American Journal of Sciences, 153, 51-63.

Wilson, M.A. \& Palmer, T.J. 1988. Nomenclature of a bival- ve boring from the Upper Ordovician of the midwestern United States. Journal of Paleontology, 62, 306-308.

Winckworth, R.A. 1930. Some new names for British marine bivalves. Proceedings of the Malacological Society of London, 19, 14-15.

Yochelson, E.L. 1999. Scaphopoda. In: Functional Morphology of the Invertebrate Skeleton (Ed. E. Savazzi). Wiley and Sons, Chichester, 363-367.

Manuscrito recibido: 15 de Noviembre, 2007 Manuscrito aceptado: 10 de Julio, 2008 